citations were identified. By expanding the search with one thesaurus term so it read: ((MRI or explode 'MAGNETIC-RESONENCE-IMAGING/all subheadings') and schizophrenia) 233 additional records were identified. Many of these would have been of considerable interest to the reviewers. We did not test the added value of other thesaurus terms such as 'NUCLEAR-MAGNETIC-RESONANCE/all subheadings'. By making the schizophrenia part of the search more sophisticated, using a published phrase for identifying Medline schizophrenia studies (Adams et al, 1998), 182 more records were identified. This subset has a high false positive rate but there are, quite clearly, citations of direct interest for a comprehensive MRI meta-analysis. When a similar exercise was undertaken on EMBASE an additional 1716 unique records were identified. Again, the false positive rate was high but there were studies of relevance to Lawrie \& Abukmeil's review. We did not investigate other rich sources of data such as PsychLit and Biological Abstracts. It is unlikely that the hand-searching of journals and references would have picked up most of the studies.

In such reviews being comprehensive is desirable. Studies that are readily accessible by simple searches on Medline may well have systematically different results to those that are more difficult to find (Egger et al, 1997).

Adams, C. E., Duggan, L., Wahlbeck, K., et ol (eds) (1998) Schizophrenic Module of the Cochrane Dotabose of Systematic Revews (updated 4 December 1997). Available in The Cochrane Library (CD-ROM). issue I. Oxford: Update Software

Egger, M., Zellweger-Zahner, T., Schneider, M., et al (1997) Language bias in randomised controlled trials published in English and German. Loncet, 350, 326-329.

Lawrie, S. M. Abukmell, S. S. (1998) Brain abnormality in schizophrenia. A systematic and quantitative review of volumetric magnetic resonance imaging studies. British journal of Psychiatry, 172, 110-120.

C. E. Adarns, B. Thornley, C. Joy Cochrane Schizophrenia Group, Middle Way, Summertown, Oxford OX2 7LG

\section{What counts as clinical research?}

Sir: Morlino et al (1997) report findings on the extent of basic research papers published that they regard as "at variance with the conclusions of Pincus et al (1993)". The difference might be related to how 'basic research' is defined. In our study, 'basic biological research' included animal studies and other research reports not involving clinical populations. Many of the papers Morlino et al included as "basic research topics such as neurochemistry, neuroanatomy and brain imaging" are likely to have involved clinical populations and probably would have been included in our category of 'clinical psychobiology'.

It is important to be clear in the use of such terms as 'clinical' and 'basic' research. The National Institute of Health's highlevel committee to review issues and problems in clinical research spent a great deal of time and effort to develop a standard definition of clinical research (National Institutes of Health Director's Panel on Clinical Research, 1997). Their definition (which engendered some controversy) includes, in addition to epidemiological, behavioural and health services research studies, "patient oriented research" that is not only conducted with human subjects but also with "material of human origin ... in which it is necessary to know the identity of the patients". Thus, 'basic' research would be limited to animal research and in vitro studies utilising human tissues that do not require dealing directly with patients. With these fairly broad criteria they found, overall, that $27 \%$ of National Institute of Health grants met the clinical research definition.

Morlino, M., Lisanti, F., Gogliettino, A., $\propto$ ol (1997) Publication trends of papers on schizophrenia. A 15 -year analysis of three general psychiatric journals. British journal of Psychiatry. 171. 452-456.

National Institutes of Health Director's Pand on Clinical Research (1997) Report to the Advisory Committee to the Notionol institutes of Heolth Director. Bethesda, MD: NIH.

Pincus, H. A., Henderson, B., Blackwood, D., et of (1993) Trends in research in two general psychiatric journals in 1969-1990: research on research. Americon journal of Psychiatry. 150, 135- 42.

H. A. Pincus American Psychiatric Association, 1400 K Street, N.W. Washington. DC 20005, USA

\section{Rapid intravenous detoxification in heroin addiction}

Sir: Despite its short follow-up period, the study by Seoane et al (1997) gives us two crucial pieces of information on the vexed question of accelerated detoxification from opioids. First, the degree of sedation in randomised groups yielded no difference in abstinence rates a month after the proce- dure. Second, the occurrence of serious side-effects was just under $5 \%$ in both the lightly and heavily sedated groups. These reactions included respiratory depression, bradycardia, pneumonia and fever of unknown origin.

Seoane et al's assertion that the incidence of side-effects is lower than with 'conventional' detoxification is only supported by reference to another 'rapid' detoxification series with a complication rate of $5.8 \%$. Traditional detoxification is believed to have a complication rate close to zero.

The prior use of methadone was not revealed. Despite being based on work done prior to 1994, there is no follow-up of data beyond four weeks. This is surprising considering the novelty and controversial nature of the treatment. A study of accelerated opioid detoxification under anaesthetic showed that $43 \%$ of patients who could be contacted had ceased their prescribed naltrexone and returned to daily heroin use at 18 months' follow-up (Rabinowirz et al, 1997).

Seoane, A., Carrasco, 6., Cabro, L., et of (1997) Efficacy and safety of two new methods of rapid intravenous detoxification in heroin addicts previously treated without success. British journol of Psychiatry; 171 340-345.

Rabinowitz, J., Cohen, H., Turrasch, R., et al (1997) Compliance to naltrexone treatment after uttra-rapid opiate detoxification: an open label naturalistic study. Drug and Alcohol Dependence, 47, 77-86.

A. Byrne 75 Redfern Street, Redfern, New South Wales 2016, Australia

\section{Moclobemide in social phobia}

Sir: The claim made by Schneier et al (1998) that moclobemide is not indicated as a first-line therapy in social phobia should be challenged. Social phobia is a relatively common anxiety disorder, which rarely presents to psychiatrists even when there is marked impairment in occupational and social functioning (Weiller et al, 1996). Thus, a first-line therapy for social phobia should be effective, well tolerated and suitable for prescription within primary care.

Addressing the latter two issues, moclobemide has a simple dosing regime and is well tolerated; Schneier et al found eightweek drop-out rates were $24 \%$ on moclobemide $\nu .25 \%$ on placebo. Their most serious objection to the use of moclobemide 